\title{
Wiki-based Community Collaboration in Organizations
}

\author{
Osama Mansour \\ Linnaeus University, Sweden \\ osama.mansour@lnu.se
}

\author{
Mustafa Abusalah \\ Consolidated Contractors \\ Company, Greece \\ mabusalah@ccc.gr
}

\author{
Linda Askenäs \\ Linnaeus University, Sweden \\ linda.askenas@lnu.se
}

\begin{abstract}
Social media technologies are increasingly used within organizational settings. Particularly, organizations continue to adopt and use wikis for enabling collaboration among their professional communities of practice. At this respect, the current paper reports results from an interpretive case study focusing on the use of a wiki for knowledge collaboration and sharing at a large multinational organization. It examines how the wiki is used by members of several professional communities of practice through interviews, observations, field studies, and documents. It concludes by showing that the openness of the wiki has a dual impact on wiki collaboration and also discusses how the wiki might serve as both an enabler and inhibitor for community and knowledge collaboration.
\end{abstract}

\section{Keywords}

Community, Wiki, Collaboration, Openness, knowledge sharing, Organizations.

\section{INTRODUCTION}

The impetus for organizations to use social media has been increasing recently. This is because the growth of social media has become a major technological evolution that transforms the way individuals and groups work and interact together [12]. Accordingly more organizations are adopting and using different social media tools at the workplace to enable collaboration and knowledge sharing amongst their employees ([36], [17]). The wiki as one type of social media is defined as a simple dynamic web page that is open for anyone to share and discuss personal knowledge in a collaborative manner. As such, the wiki is increasingly used for different internal knowledge management purposes in organizations ([36], [9], [30]). Most often, the wiki is used in organizations by virtual communities of practice (CoPs) [36], which are central to knowledge management strategies [3]. For instance, Hasan \& Pfaff [10] and Wagner [28] described the wiki as a conversational knowledge management tool that is used by CoPs to address specific knowledge needs. Given its flexible nature, the wiki has also been described as a lean approach to web-based content management allowing multiple users to collaborate for the creation of content [9].
Further, a study by Stenmark [22] shows how the use of the wiki has enabled more participation and knowledge sharing in an organization that wanted to activate its intranet.

Yet, despite the recognition by managers of the value of knowledge ([19], [34], [35]) and the need to develop knowledge management strategies in their organizations, they are still unclear about how to do that [34]. Several attempts by organizations to use information systems in order to manage their knowledge have resulted digital junkyards [34]. Stocker et al. [24], for instance, found that organizations using wikis struggle with the dilemma of a knowledge-sharing environment. While some managers perceive higher benefits from using wikis to transfer and manage knowledge [24], others tend to be uncomfortable with the idea that their content is open and accessible by large numbers of users [30]. In the same vein Danis \& Singer [8] argued that the uncoordinated use of wikis by many users may result chaos such as distrusted content, difficult navigation, and inconsistency among wiki pages.

As the number of organizations introducing and using wikis is growing, still only a little amount of empirical knowledge is available about this phenomenon ([5], [24], [8], [23], [17]). In contrast, many empirical studies examining wikis are often found in other contexts mainly in education and Wikipedia, which are fundamentally different contexts than the workplace ([24], [8]). Given the paucity and need of an empirical understanding of using wikis in an organizational context and the importance of this emerging phenomenon, the current paper focuses on the use of the wiki for knowledge collaboration and sharing by several CoPs at a large multinational organization. It explores and examines the perceptions of senior employees who serve as community managers and captains in different professional wiki-based communities and seeks an answer of how they react and behave towards the introduction and use of the wiki. As such, the paper is based on an interpretive exploratory study including multiple sources of data such as interviews, field notes, documents, and observations. The ultimate aim is to identify and explain factors that influence the use of the wiki as a shared medium for community and knowledge collaboration within organizational settings.

Hence the unique contribution of this paper is providing novel understandings about using wikis in organizations. Mainly the paper contributes rich insights of wiki openness and its influence on how communities use a wiki for knowledge collaboration and sharing at the workplace. 


\section{RELATED LITERATURE}

\section{Wikis}

The first exposure to wikis was in 1994 by Ward Cunningham who wanted to have a platform for software developers and designers to collaborate and share knowledge ([22], [23]). A wiki, a Hawaiian word means quick, refers to a simple dynamically updated web page that is open for anyone to add, edit, discuss, and track content. It consists of hyperlinked pages that allow anyone to collaborate openly for the creation and modification of knowledge ([36], [9], [19]). One of the unique attributes of wikis, which differs from pervious technologies, is the free or open editability that enables anyone to edit others' contributions in order to improve readability, organize pages, and integrate ideas [36]. In a similar sense, Yates et al. [36] referred to the process of rewriting, reorganizing, and integrating wiki content as "shaping". The defined shaping as a purposeful activity that transforms existing knowledge on the wiki into useful knowledge. Nowadays one of the most famous examples of the wiki concept is the large online encyclopedia called Wikipedia [9]. Wikipedia well represents the concept of open wiki collaboration, as it is an outcome of the many voluntary contributions made by Wikipedians ([11], [31]).

\section{Wikis in Organizations}

Perhaps the term that best describes the use of wikis in organizations is Enterprise 2.0. Andrew McAfee [18] coined this term to represent organizations that build and use social media or web 2.0 technologies namely wikis, blogs, and others at the workplace. The use of wikis in organizations is far different from how it is used in other contexts such as educational settings or open environments like Wikipedia ([8], [23]). Many scholars (e.g. [11], [20], [29], [10], [28], [16]) discussed several possibilities of using wikis within organizational settings. For instance, Majchrzak et al. [17] conducted a survey focusing on the corporate use of wikis. They provided a list including several activities supported by the use of the wiki such as communities of practice and user groups, ad-hoc collaboration, e-learning, project management, etc. Others such as ([10], [28], [29]) described wikis as conversational knowledge management technologies. A wiki as a conversational knowledge management tool represents an end-user developed approach that is based on collaboration and conversation ([10], [29]). In the same vein, Kosonen \& Kianto [16] argued that wikis represent a socio-technical approach to managing knowledge that enables a multitude of knowledge-work processes, combines communication and personal information management, and makes knowledge work more visible. However, Danis \& Singer [8] argued that the open nature of the wiki might introduce difficulties for organizations, for instance, to manage their content, which may result chaos, inconsistency, and difficult navigation. Other scholars discussed trust problems [14] and vandalism and malicious edits [9] of wiki content due to its free, open nature. In the following section we describe how a wiki is used by Communities of Practice.
Wikis for Community Collaboration and Knowledge Management

Wikis are increasingly used in organizations by virtual communities of practice [36]. In this respect, Ardichvili [3] explained that CoPs are gaining popularity as a vehicle of collective learning and knowledge creation within organizations. A community of practice (CoP) is defined as a group of people informally bound together by shared expertise and passion for a joint enterprise, who deepen their knowledge and expertise by interacting on an ongoing basis ([32], [34], [33]).

Wenger \& Snyder [33] explained that CoPs differ from other forms of organization e.g. project teams or formal work groups in terms of purpose, belonging, and bond among community members. The purpose of CoPs is to exchange knowledge and develop capabilities. Members of the community select themselves to participate with others, and passion and commitment are bonds that hold them together. Ardichvili [3] explained that one of the most recognized benefits of CoPs is their ability to allow for the generation and dissemination of tacit knowledge, which is hard to communicate, as it is intuitive and embedded in a specific context. He referred to CoPs as a platform for sharing and internalizing tacit knowledge. Within organizational settings, Ardichvili [3] further argued that CoPs play a central role in the knowledge management strategy. However, Wenger [32] explained that CoPs need a technological infrastructure that enables members to communicate regularly and accumulate documents.

In this respect, Knowledge Management Systems (KMSs) refer to a class of information systems applied to managing organizational knowledge. They are IT-based systems developed to support and enhance organizational knowledge creation, storage, retrieval, transfer, and application [2]. As such a wiki represents one kind of KMSs that allows members of communities of practice to create, share, and aggregate their knowledge into a new intellectual and organizational capital [36]. Wagner [28] explained that conversational knowledge creation using wikis emerged as the most popular way for organizations to create knowledge in the context of online communities. Accordingly wikis can be used for several collaborative activities. For instance, Majchrzak et al. [17] reported that organizations might improve their collaboration, work processes, and knowledge reuse using wikis. Also experienced organizational members might gain reputational benefits from sharing their experiences with others. Yet, organizations are often faced with several challenges when using wikis. For instance, Happel \& Treitz [9] discussed wiki proliferation that represents several side effects of wiki growth such as old and outdated content, redundant information, and poor linkage between content. They argued that such problems might result a lack of acceptance of the wiki by new users and loss of existing users. Also, Yates et al. [36] explained challenges for understanding the motivation for knowledge shaping that is critical to maintain the evolution of knowledge on a wiki. 
As the modern economy runs on knowledge ([33], [35]), knowledge management is considered one of the major sources of competitive advantage in modern organizations [28]. Knowledge management is defined as the process of identifying and leveraging the collective knowledge in an organization for competition purposes [2]. Knowledge, in the sense of communities of practice, is an accumulated outcome of the ongoing process of exchanging and contributing knowledge to the community [32]. Other scholars have discussed different perspectives of knowledge (e.g. [13], [2]). Wenger [32] viewed communities of practice as the social fabric of knowledge and argued that $\mathrm{CoPs}$ are the cornerstones of knowledge management. He identified three characteristics of CoPs that represent the foundation of a knowledge strategy in an organization. First, domain, which is the area of knowledge that brings the community together, gives it its identity, and defines the key issues that members need to address. Second, community, which is a group of people for whom the domain is relevant and involves members who interact and develop relationships that enable them to address problems and share knowledge. Third, practice, that is the body of knowledge, methods, tools, stories, cases, documents, which members share and develop together. The combination of these three characteristics is what enables CoPs to manage knowledge. At this respect, wikis as an end-user approach can enable the combination of these characteristics by allowing members of CoPs to jointly create and share common knowledge and experiences [29]. Hence a wiki as a free, open technology may provide a flexible platform that helps community members engage in voluntary collaboration and enable dynamic interactions among them. The remaining parts of this paper focus on examining the wiki environment and explain factors influencing wiki collaboration.

\section{RESEARCH APPROACH}

In this research we chose to be explorative since only a little or no research aimed at empirically examining the perceptions of managers of using wikis in organizational settings. In other words, the paper aims to explore the phenomenon of using wikis in organizations inductively. This choice is stimulated by both the nature of the problem being a new and complex social phenomenon and the likely high-degree of its uncertainty due to the paucity of empirical knowledge [25]. Thus the study adopts an interpretive approach to research. It emphasizes, in a phenomenological sense, that an interpretive understanding of human experiences can be derived from data collected in real-life settings [21]. As such, the process of collecting and analyzing empirical data is informed by this interpretive philosophy that aims to produce a deeper understanding of the phenomenon as given by the participants ([27], [6], [26]). An in-depth exploratory case study research strategy has been the vehicle of our process of inquiry. It is used to help us in capturing interpretations and meanings that our participants assign to their interactions as a community in real-life settings [26].
Such an approach would help us in obtaining a clear and deep understanding of the perceptions and attitudes of our participants by uncovering recurring processes and meanings of their ongoing human activity. In the following sections we present and discuss the context of our case as well as the processes of data collection and analysis.

\section{Description of the Case}

The study took place at Consolidated Contractors Company (CCC), a large multinational contracting organization, which has over 160,000 employees distributed all over the world. The organization is headquartered in Athens, Greece and has offices in the five different continents. Teams of senior and junior employees including project managers, mechanical engineers, technicians, etc. perform a variety of civil and mechanical construction projects such as building harbors, airports, tunnels, and Gas and oil plants in different contexts. These teams might work in the middle of the sea or the desert. The size of these teams may vary depending on the size of projects ranging from 2000 employees in smaller projects up to 30,000 employees in larger projects.

Due to this distributed nature of the organization and the dispersion of project teams, top management started to think of how to leverage and manage dynamic knowledge and experience of such a vast number of employees. It is worth mentioning that the company has increased its employees in the last ten years from 35,000 up to 160,000 . This explosion in the number of employees has further stimulated top management to think about flexible ways for capturing and managing knowledge and experience at CCC. In this respect, CCC mainly used a document management system for storing and organizing its knowledge into structured documents and reports. This system was ineffective to allow for dynamic collaboration and sharing of knowledge and experiences. Consequently, top management has decided and supported the establishment of a Knowledge Management (KM) department that is responsible for developing and managing a shared platform for collaboration and knowledge sharing at CCC. Thereafter, the KM department was officially established as of July 2007. The KM department, after eight months of planning, launched the wiki, which is called 'Fanous' that is an Arabic word means "The Lantern" in March 2008.

In order to put the wiki into operation, the KM department established a core team of senior employees and top managers. This team represented well-experienced organizational members who have been working at CCC for a long time. The team aimed at providing a basis for building and cultivating different specialized communities as well as promote the use of the wiki amongst their employees. Then the wiki started to operate with five professional communities of practice $(\mathrm{CoPs})$, as they refer to them in the company. Each community is specialized in a particular domain and is led by a community manager and a number of community captains. Also there are Subject Matter Experts (SMEs) who are expert employees within the domain of the community. 
All these members are selected based on their seniority and level of experience with full accessibility to add, edit, comment, and change contributions on the wiki. Other people at the company can access the wiki but with roles limited to reading and commenting on the articles due to control measures. Each community has its own space on the wiki that includes community pages where community members collaborate and share knowledge with each other. Members can also contribute to other relevant communities on the wiki. In addition, all $\mathrm{CoP}$ members receive daily notifications to keep them updated of any new contributions. In 2009, the wiki included $10 \mathrm{CoPs}, 700$ active members, and 3237 contributions.

\section{Wiki as a Knowledge Management Tool at CCC}

Document Management Systems represent a traditional approach of storing, organizing, and searching for organizational knowledge at CCC. This knowledge can be accessed based on access rights criteria. Yet, the adoption of an innovative knowledge sharing platform requires a collaborative, social oriented medium that facilitates flexible knowledge collaboration and sharing. In order to satisfy this aim, the KM department decided to use a wiki as a collaboration knowledge management tool.

The wiki consists of several spaces that belong to different CoPs. Members of these CoPs are distributed across different regions and projects within a particular field and they use their wiki space to collaborate, explore ideas, and discuss work problems. The advantage of using a wiki over other collaboration tools such as forums is that all $\mathrm{CoP}$ members can edit articles published by other members; so more than one member can collaborate to prepare an article. Also contributors can track changes of their articles through wiki versioning. This practice is very important as it provides a dynamic basis for CoPs to collaborate in order to produce method statements that describe best practices, work flows, work procedures, etc. [1]. As we discussed before in the introduction, uncoordinated contributions to the wiki may result chaos. To address this challenge, the wiki was designed based on spaces. Each space is used by a particular CoP and is organized hierarchically based on areas and topics related to that CoP. Any new contribution will be categorized under the related topic. This method of categorization allowed community members to easily navigate and locate contributions. Further, the wiki contains contributions that are collaboratively created by more than one author while other contributions are based on personal or organizational experience and contributed by a single author. To audit the quality of contributions and to inspire additional coauthoring and editing, the KM department employed "content review workflow", which was implemented after the completion of this study, to produce high-quality contributions. This does not mean that some contributions are void or invalid. But, for instance, two different contributors might author the same method statement in two different ways. When a user accesses the wiki to search for this method statement, he wants to pick up the best practice out of these two method statements.
Captains of each CoP can use the "content review workflow" to obtain best practices and to ensure the integrity of contributions. In addition, the wiki is semi moderated so that members can only author articles that are related to work. Yet, it is very unlikely that a comment or an article is deleted from the wiki due to lack of relevance.

\section{Data Collection Process}

The selection of participants for our research marks the early stages of the data collection process. The main criteria for selecting our sample include the seniority level, membership in different wiki communities, level of activity, computer skills, etc. We used emails to send invitations to several employees who match our criteria. These emails contained general information about the purpose of the study and other practical information related to the interviewing process such as voluntary participation, privacy and confidentiality issues, interviewing time, etc. The resulted sample of participants who responded to our emails was twelve self-selected senior employees and managers with a range of experience between ten years up to thirty years. Many of these participants have a three-year experience of using the wiki and serve either as community managers who lead the community, or community captains who are active wiki users in suggesting topics, monitoring contributions, encouraging members, etc. in different specialized CoPs in the wiki environment. Only a few others are lurkers who may read the wiki quite often but seldom make any contributions. Further, most of our participants hold university degrees ranging from a Bachelor up to the Ph.D. mainly in construction and engineering related subjects. In respect to computer experience, many of our participants have modest computer knowledge with varied experiences in using social media technologies such as Facebook, Wikipedia, and LinkedIn. By and large, participants in this research represent seniors with varied levels of activity in the wiki. When the wiki was first introduced, most of these seniors were selected by the KM department to be part of a core team in order to motivate a "grassroots" initiative for using the wiki at CCC. This particular sample of senior participants was important to understand evolving perceptions of using the wiki since its introduction.

The primary vehicle for our data collection was the in-depth semi-structured interview. However, we have triangulated multiple sources of data including interviews, field notes, organizational records, and participant observations. The triangulation of multiple sources of data has been useful to address a broader range of behavioral issues [37] and increase the robustness of our data [21]. Given the geographical distribution of our participants in different parts of the world, ten interviews have been conducted either via telephone or an online conferencing system e.g. Skype. Only two face-to-face interviews have been conducted during the first field visit. The average interviewing time was one hour and all interviews have been recorded and transcribed for later analytical purposes. A case study protocol has been used to guide us throughout 
the data collection [37]. This protocol included a general overview of the study and also several themes and issues to frame our questions and discussions with the interviewees. These themes have been mainly developed based on the literature of the wiki technology (cf. sections 2.1, 2.2, 2.3) and also through our preliminary discussions with KM specialists about the wiki platform. For instance, themes related to the openness and free editability of the wiki have been the basis for asking questions about their impact on collaboration and knowledge sharing at the workplace. Instead of using a pre-defined set of questions, these themes allowed us to freely explore the perceptions of our participants and also discuss other emergent issues during the conversation. In other words, we used a fluid stream of questions rather than rigid to follow the line of inquiry [37].

Further sources of data were obtained during two field visits at the company. The first visit was at $\mathrm{CCC}$ headquarters in Athens, Greece and the second was at CCC offices in Abu Dhabi, UAE. During the first visit we carried out an observation of the wiki platform with the help of the second author who works at the KM department. Our observation of the wiki has helped us to get deeper insights of how different communities are structured and categorized into specialized areas and also understand how community members interact with each other. Also, field notes have been taken both during the observation of the wiki and our discussions with the head of the KM department and other KM specialists. These notes mainly contained information about issues and challenges related to the wiki such as how the wiki works, the introduction of new communities, sustaining the current communities, etc.

The second visit aimed at participating in the $10^{\text {th }}$ quarterly meeting of the 'hydro testing and precommissioning' community. Our role as participant observers was useful to obtain an insider view of these meetings [35] as we have been observing actual discussions and interactions among community members while exchanging ideas and experiences related to the content of their community on the wiki. In addition, we have obtained several electronic volumes of the monthly newsletter published by the KM department. The newsletter includes a variety of information related to community updates, featured articles from different wiki communities, and monthly statistics about top contributors and contributions This information was a useful resource to provide us with additional insights of communities' activities.

Data Analysis and Validation

Perhaps the concept of hermeneutic circle discussed by Klein \& Myers [15] best describes the process of analyzing our collected data. The concept of hermeneutic circle is foundational to all interpretive work [15]. It emphasizes that a whole understanding of the phenomenon can be achieved through circular understanding and interpretation of its parts and their interrelationships ([7], [15], [4]). Based on this, each interview transcript was reviewed and several segments or parts have been identified and examined.
Then we started to go back and forth across the text in order to create connections among these parts in order to develop a whole understanding. While moving through a circle of understanding we have been able to identify several interconnected perspectives and themes that explain the phenomenon. Both open and axial coding were combined to drive this circle of understanding through identifying the themes and their relationships [21]. For data validation, a number of random interview transcripts have been send to the interviewees who agreed to receive them in order to validate the conversation and revise their views. We have been able to receive their views that the transcripts well represent their perceptions of the wiki. No modifications have been requested or made by the interviewees.

\section{FINDINGS}

Our research findings provide rich insights of how organizations use the wiki technology to enable and support collaborative practices such as gathering and sharing knowledge by their individuals and communities. Our participants described the wiki in synonym terms with KM as one of the Group Technical Managers explained the role of the wiki in managing knowledge at CCC:

"The basic principle of KM first of all is gathering of experience gained by the people in the company, which until KM was introduced, was the property of this individual and it was not spread. And the second step of $K M$ was the systematic analysis of the subject and the spreading of this knowledge to selected users. So this is the only vehicle, you cannot spread to such vast number of users such information by any other means".

In this respect, $\mathrm{CCC}$ uses the wiki for specific aims and objectives, which makes it a formal tool for managing and sharing work-specific knowledge. A Control Project Manager said:

"...our knowledge management is very specific to $C C C$, and very applicable towards our own procedures."

Also a Mechanical Construction Manager explained:

"We are not general users of the wiki, we have an aim from the wiki to use it efficiently and effectively for our work and socially for our community, for CCC community"

The Group Technical Manager provided an example from his community that focuses on pipe fabrication and technology improvements. He explained:

"...there are already about 620 entries and these entries maybe specifications, maybe specifically written articles by the participants or by others ... they found the subject very interesting and participated with their long experience."

The wiki is often used by members who belong to several Communities of Practice (CoPs) to share communityspecific experiences and knowledge with each other and collaborate for solving mutual problems and proposing solutions related to their work. One of the Control Project Managers described this through his experience in the mobilization community as follows: 
"it's much easier now, if I mobilize to a new area, I can easily go to this COP, community of practice, for mobilization to remote areas, and I can access a lot of information, and it's not only this, I can share my problems with my colleagues on the other side of the globe."

Further, the wiki is also used to help people connect with each other and establish relationships with experienced community members:

"...it will give me an opportunity to know more about these people, what are their titles, or functions within the project or the company. So it is introducing more people through this media instead of just sitting and knowing the persons around you".

Open and transparent interactions among community members through the wiki was perceived by many of our participants to be one important mean to connect with other employees and locate experienced members. A Group Quality Manager said:

"...the wiki itself have brought all the experts closer in the community. What I mean, now we know who is the expert in our domain, whom we can talk to about a particular issue...Now we understand that we belong to a community..."

However, the way the wiki is used by community members was influenced by various factors mostly related to the open nature of wiki collaboration within communities. For instance, the voluntary nature of a wiki that allows anyone to openly and freely contribute and edit others' contributions was conceived by our participants as one of the barriers for wiki collaboration. As such, a Construction Manager said:

"...it is not a formal tool to be utilized as a sort of communication ... it is not that much official source that I get something related directly to my job and take it".

Also, the idea of an open wiki where knowledge is accessible by anyone at the company was a major concern for many of our participants. One of the Group Plant Managers explained his stance towards an open wiki:

"I did not support that such thing when it falls in the hands of others will make us less competitive, I totally disagreed with that".

Other participants had a different stance and explained that a wiki needs to be uncontrolled and open for everyone in order to enable flexible and dynamic community collaboration. In this respect, the head of $R \& D$ for open source development said:

"...you need to create an uncontrolled space and you just allow people to go and talk ... if you want to make it formal, people will not talk, you need to make it really informal'.

Further issues related to wiki openness show that it has an influence on the willingness of people to contribute and share with each other through the wiki. On the one hand, a Construction Manager explained how wiki openness might cause him problems if he comments or edits contributions by an upper management person:

"I don't want to edit for him in front of many users, they will see that I already attended his article ... he will consider that an insult in front of others".

On the other, a Mechanical Construction Manager explained that the wiki has positively influenced his contributing and sharing behavior. He said:

“...for my part, I feel it; I have more interest ... in giving information. I really feel my information is very valuable when I put it on the wiki, and people are looking at it".

A Mechanical Manager generally reflected his view of the openness of the wiki as follows:

'Some people don't share because they don't like their ideas to be discussed openly on the wiki because anyone can open the it and see the discussion. I believe that those people don't have enough experience; anybody who has confidence in himself will participate in the discussion."

In the same vein, the openness of wiki collaboration stimulates the effect of the community in the sense that when community members see others contributing, they feel more motivated to do the same. A Proposal Leader said:

"When you see more people participating, when you see more people writing, when you feel more confident that the people who will read your input know what you are talking about you start to be more cooperative. I think this is what added and improved my perception".

While many of the above issues can be seen as enablers for wiki collaboration, challenges also arise because of the openness of the wiki. For instance, a Group Plant Manager expressed his opinion about making knowledge open and accessible by anyone at the company as follows:

"I was really against such thing that we just fill pages because you know people simply would like to show their contributions, quantity is sick sometimes".

This statement reflects concerns about the quality of contributed knowledge on the wiki since people might only contribute to show off in front of a large number of users. A Group Quality Manager commented on this:

"the more contribution on a particular topic, the other members of the community, they treat you as being the expert in this field. This is not necessarily true (laugh) because what we have to take into account is the quality of the contribution. Quantity can be huge but quality could be very low".

Such concerns have encouraged controlling contributions on the wiki. The Group Quality Manager explained:

"Once a piece of knowledge is submitted, a document for instance, it will be will be submitted to the knowledge expert who will review it and he will have to say yes or no, to put it on the wiki or not. The wiki will have only the validated knowledge available for the user". 
In addition, each community has regular meetings for its members where they can discuss and agree on several issues related to their content in the wiki environment.

"...it is not only exchanging ideas online, we have meetings, we go and people of these communities meet and discuss things, and the thing is this is some kind of filtering and coming up with better ideas and coming up with consensus and agreement of these ideas, so its not only writing and reading"

\section{DISCUSSION}

Wikis for Community Collaboration and Knowledge Sharing Our findings reveal that the wiki is used for open collaboration and knowledge sharing but to some extent in a controlled manner. There is a mixed attitude towards the use of a wiki for open collaboration as many of our participants explained that the wiki should be open for all but at the same time some rules of control such as monitoring and reviewing contributed knowledge should be applied. Mainly, this is because the organization leans toward a formal use of the wiki with specific and clear objectives in order to ensure a reliable application of the wiki at the workplace. This situation can be described as a kind of controlled openness. In the same vein, our participants strongly expressed their satisfaction with the wiki as a dynamic medium that allows knowledge and experience to be accessible by a large number of people at the company. We also noticed that the nature of the wiki as an open and voluntary tool was useful to stimulate people to share their knowledge and experiences as well as enrich their sense of belonging and responsibility to the community cf. [33]. This is an intriguing aspect of the wiki especially when compared to other types of collaborative technologies in organizations. The wiki has given experienced people the chance to share their experiences and make it accessible to a large number of people for the benefit of the organization. In addition, by providing communities with a shared place, the wiki has enabled community members to connect with each other and identify who the expert is within a particular community and therefore strengthens their attachment and belonging to the community as it becomes a source of relationships with experienced members and useful knowledge to their work.

\section{Behavioral Issues in Organizational Wiki-based Community} Collaboration

Behavioral issues of using the wiki for open management and sharing of knowledge were salient throughout our discussions with several managers. These issues have a considerable impact to some extent on the use of the wiki for community collaboration and knowledge sharing. As reported by other scholars (e.g. [36], [3], [35]) several barriers and motives might influence the contributions of people to communities of practice. In this respect, we found further issues related to barriers and motives of using and contributing to the wiki by CoPs. The openness of the wiki has a dual impact on knowledge collaboration and sharing by community members.
For instance, the open nature of wiki collaboration might deter people from contributing and sharing their knowledge, even if they are willing to do so, because they are not comfortable with exposing themselves to the public cf. [30] or an unknown audience. These people have two characteristics. First they might be a kind of people who do not accept criticism or might not accept the comments made by others to edit or shape their contributions cf. [36]. Second it might be that they feel shy to expose themselves to a large number of people or prefer personal communication as a personal trait. Related to this, there is a lack of confidence and courage to comment on contributions made by higher-level contributors, as people do not feel comfortable to publicly discuss or comment on issues contributed by senior people who are higher in rank and experience. As a result hierarchy and ascendancy are carried out to the wiki environment and might serve as barriers to wiki collaboration. Also, the assumption that people might take the opportunity to contribute in order to be proud of themselves in the open environment was conceived to be a threat to the quality of contributed knowledge. Further, the voluntary and informal nature of wiki collaboration can also be a barrier to share and contribute to the community in the sense that people do not see the wiki as part of their jobs. At this respect, a number of our participants expressed the need for organizational pressure to consider the wiki as a required tool at the workplace.

In contrast, while the openness of the wiki has created several barriers to collaborate and share, it has also attracted people to freely express themselves and openly collaborate and share their knowledge with others. This has made it easier for the community to access knowledge and locate experiences. More important, people feel that their knowledge is more valuable when it is open for others who read it and then use it in their real work, which therefore provides them with extra motivation to collaborate and share with others. Moreover the openness of the wiki was an important factor to stimulate the effect of the community within community members. So when people see others contributing and sharing they become motivated to do the same which to some extent reduces the constraining effect of hierarchy and increases collaboration and sharing. In the same vein, we found that open wiki collaboration has enriched the sense of the community at the organization. The openness of wiki collaboration has made people closer and more connected in the sense that they can socially interact with each other and meet new people who might be experts in relevant areas. Consequently, the wiki is considered as a source not only for knowledge but also for relationships with knowledge contributors, which might emerge during open discussions and commenting on the wiki among community members.

In respect to quality issues, the openness of the wiki was not considered a threat on the quality of contributed knowledge by many of our participants who are in favor of a controlled wiki environment cf. [10]. 
While many of them explained their concerns about these issues, being an internal and controlled environment that is only accessible, in the sense of being able to change and edit content, by selected experienced organizational members made the wiki a secure medium for sharing reliable and trusted knowledge. At this respect, there are many forms by which the organization controls the wiki such as monitoring and reviewing contributions by community managers and captains as well as KM specialists, discussing contributions during community meetings, and defining various accessibility measures. In other words, there is a level of control applied to the use of the wiki in organizational settings, which limits any potential quality problems that might exist because of its openness and free editability. In addition, the sense of responsibility by experienced organizational members has driven openness at the company in the sense that the wiki has given them a chance to make their experience public and accessible by everyone. Once this experience is available on the wiki, the nature of work, which requires employees to look for different procedures and methods necessary to do their jobs, drives them to join wiki communities in order to access and benefit from this experience. Both the nature of work and the sense of responsibility are driven by open wiki collaboration but are also vehicles for openness.

\section{CONCLUSIONS AND FUTURE WORK}

This paper aimed at examining and explaining factors that influence the use of the wiki for knowledge collaboration and sharing by members of communities of practice within organizational settings. In this respect, the openness of the wiki was found to be one major factor that has a dual impact on determining peoples' behaviors and attitudes towards the use of the wiki at the workplace.

As such, the open nature of wiki collaboration was both a barrier and an enabler for community members to collaborate and share with each other. The hindering impact of openness can deter or lead to less collaboration that may result from the lack of comfort by people to openly contribute and share their knowledge in front of a large number of fellow employees or an unknown audience. This might be caused by the fact that these people are not willing to accept others' comments and edits or they feel more comfortable with personal and less disclosed communication. Also, hierarchal constraints are carried out to the wiki environment, which may prevent people from editing and commenting on articles by their superiors in public.

In contrast, the enabling impact of openness has helped in attracting more contributors and the creation of new relationships among community members through the effect of the community that results from transparent interactions. Also, the open accessibility of knowledge has a positive impact on knowledge contributors in the sense of feeling that their knowledge is more valuable especially when others can read and use this knowledge.
Understanding this dual impact of wiki openness is increasingly important as more organizations are adopting wikis at the workplace. This importance stems from the fact that openness as a major wiki property may have a determining impact on the success or failure of implementing a wiki as a medium for collaborative practices in organizations. Therefore more research is needed to further examine the duality of wiki openness and the eventual enactment of paradoxical organizational structures and cultures. It is also recommended to conduct longitudinal studies that focus on examining gradual behavioral changes of community members in respect to open wiki collaboration.

\section{ACKNOWLEDGMENTS}

I wish to acknowledge the KM department at CCC represented by Dr. Amr El-sersy and Dr. Mustafa Abusalah for their generous cooperation and endless support for my empirical research. I also wish to thank and express gratitude to all participants in this research for sharing their precious time and insightful discussions. In addition, I thank my supervisors and all colleagues and research fellows for their support and insightful comments.

\section{REFERENCES}

1. Abusalah M. (2008). Wiki @ CCC, Consolidated Contractors Company Bulletin: Knowledge Management, (87), $2^{\text {nd }}$ Quarter 2008, p. 16.

2. Alavi, M., and Leidner, D. (2001). Knowledge management and knowledge management systems: conceptual foundations and research issues, Management Information Systems Quarterly, 25 (1), $107-136$.

3. Ardichvili, A. (2008). Learning and Knowledge Sharing in Virtual Communities of Practice: Motivators, Barriers, and Enablers, Advances in Developing Human Resources, 10 (4), 541 - 554.

4. Butler, T. (1998). Towards a hermeneutic method for interpretive research in information systems, Journal of Information Technology, 13, $285-300$.

5. Chai, S., Joseph, P., and Mullins, P. (2010). The Empirical Investigation of a Wiki based group system in organizations, In proceedings of the $16^{\text {th }}$ Americas Conference on Information Systems, Lima, Peru, August $12-15$.

6. Chen, W., and Hirschheim, R. (2004). A paradigmatic and methodological examination of information systems research from 1991 to 2001, Information Systems Journal, 14, 197 - 235.

7. Cole, M., and Avison, D. (2007). The potential of hermeneutics in information systems research, European Journal of Information Systems, 16, 820 833.

8. Danis, C., and Singer, D. (2008). A Wiki Instance in the Enterprise: Opportunities, Concerns, and Reality, Computer Supported Cooperative Work, San Diego, USA, November $8-12$.

9. Happel, H., and Treitz, M. (2008). Proliferation in Enterprise Wikis, In proceedings of the 8 th International Conference on the Design of Cooperative Systems, Carry le Rouet, France. 
10. Hasan, H., and Pfaf, C. (2006). Emergent Conversational Technologies that are Democratizing Information Systems in Organizations: the case of the corporate Wiki, In proceedings of the Information Systems Foundations (ISF): Theory, Representation and Reality Conference, Australian National University, Canberra, Australia, 27-28 September.

11. Hester, A., and Scott, J. (2008). A conceptual model of wiki technology diffusion, In proceedings of the $41^{s t}$ Hawaii International Conference on Systems Sciences, The Big Island, January $7-10$.

12. Hirschheim, R., and Heinz, K. (2010). A Short and Glorious History of the Information Systems Field, To appear in the Journal of the Association of Information Systems.

13. Jashapara, A. (2004). Knowledge management: an integrated approach, Pearson Education Limited, England.

14. Kittur, A., Suh, B., Chi, E. (2008). Can you ever trust a wiki? Impacting perceived trustworthiness in Wikipedia, Computer Supported Cooperative Work (CSCW' 08), San Diego, California, USA, November $8-12$.

15. Klein, H., and Myers, M. (1999). A Set of Principles for Conducting and Evaluating Interpretive Field Studies in Information Systems, Management Information Systems Quarterly, (23) 1, 67 - 94.

16. Kosonen, M., Kianto, A. (2009). Applying wikis to managing knowledge - a socio-technical approach, Knowledge and Process Management, 16 (1), pp. 23 29.

17. Majchrzak, A., Wagner, C., and Yates, D. (2006). Corporate wiki users: results of a survey, WikiSym '06, Odense, Denmark, August 21 - 23.

18. McAfee, A. (2006). Enterprise 2.0: The dawn of emergent collaboration, MIT Sloan Management Review, 47 (3), 21 - 28.

19. Pfaff, C., and Hasan. H. (2007). Collaborative knowledge at the Grass-roots level: the risks and rewards of corporate wikis, In proceedings of the $11^{\text {th }}$ Pacific-Asia Conference on Information Systems, Auckland, July $3-6$.

20. Raman, M. (2006). Wiki technology as a "free" collaborative tool within an organization setting, Information Systems Management, 23 (4), 50 - 66.

21. Rowlands, B. (2003). Employing Interpretive Research to Build Theory of Information Systems Practice, Australasian Journal of Information Systems, (2) 1.

22. Stenmark, D. (2005). Knowledge Sharing on a Corporate Intranet: Effects of Re-Instating Web Authoring Capability, In proceedings of the $13^{\text {th }}$ European Conference on Information Systems, Rustenburg, Germany.
23. Stenmark, D. (2008). Web 2.0 in the business environment: The new intranet or a passing hype?, In proceedings of the 16th European Conference on Information Systems, Galway, Ireland, June 9-11.

24. Stocker, A., Grantizer, G., and Tochtermann, K. (2009). Exploring the Value of Enterprise Wikis: A Multiple-Case Study, In proceedings the International Conference on Knowledge Management and Information Sharing, Madeira, Portugal, October 6-8.

25. Trauth, E. (2001). Qualitative Research in IS: Issues and Trends, Idea Publishing.

26. Walsham, G. (1995). Interpretive case studies in IS research: nature and method, European Journal of Information Systems, (4) 2, $74-81$.

27. Walsham, G. (2006). Doing interpretive research, European Journal of Information Systems, (15), 320 330.

28. Wagner, C. (2004). Wiki: A technology for conversational knowledge management and group collaboration, Communications of the Association of Information Systems, 13, 265 - 289.

29. Wagner, C. (2006). Breaking the knowledge acquisition bottleneck through conversational knowledge management, Information Resources and Management Journal, 19 (1), 70 - 83.

30. Wagner, C., and Majchrzak, A. (2007). Enabling customer-centricity using wikis and the wiki way, Journal of Management Information Systems, 23 (3), $17-43$.

31. Wagner, C., and Prasarnphanich, P. (2007). Innovating collaborative content creation: the role of altruism and wiki technology, In proceedings of the $40^{\text {th }}$ Hawaii International conference on Systems Sciences, The Big Island, January $3-6$.

32. Wenger, E. (2004). Knowledge management as a doughnut: shaping your knowledge strategy through communities of practice, Ivey Business Journal.

33. Wenger, E., and Snyder, W. (2000). Communities of Practice: the organizational frontier, Harvard Business Review.

34. Wenger, E., McDermott, R., and Snyder, W. (2002). Cultivating Communities of Practice, Harvard Business School Press, Cambridge, MA.

35. Wasko, M., and Faraj, S. (2000). "It is what one does": Why people participate and help others in electronic communities of practice, Journal of Strategic Information Systems, 9, 155 - 173.

36. Yates, D., Wagner, C., and Majchrzak, A. (2010). Factors Affecting Shapers of Organizational Wikis, Journal of the American Society For Information Science and Technology, 61(3), $543-554$.

37. Yin, R. (2009). Case Study Research: Design and Methods, London, UK. 